\title{
Fast Color-Weakness Compensation with Discrimination Threshold Matching
}

\author{
Rika Mochizuki $^{12}$, Satoshi Oshima ${ }^{2}$, and Jinhui Chao ${ }^{2}$ \\ 1 NTT Cyber Solutions Laboratories, 1-1 Hikarinooka, Yokosuka-shi, Kanagawa, \\ 239-0847 Japan \\ 2 Chuo University, 1-13-27 Kasuga, Bunkyo-ku, Tokyo, 112-8551 Japan
}

\begin{abstract}
We present a method to compensate color-weak vision along the confusion lines, based on matching between discrimination thresholds of the color-weak observer and of color-normals. The compensation and simulation map preserve color-differences of every pair of colors between color-normals and the color-weak observer. We developed an explicit formula for compensation and simulation of color-weak vision in closed form. The method is easy to implement and fast.
\end{abstract}

\section{Introduction}

The adaptation of color information for color-weak observers is one of the most important subjects in universal design and barrier-free IT technology. Currently used methods either enhance color contrast between symbols and background or need to define criteria to satisfy certain, often conflicting, visual restrictions. The former approach therefore do not apply to natural images, the latters obtain compensation colors by optimization and the solutions are not unique and difficult to justify. Besides, since these approaches avoid colors which are hard to see for color-weak observers and replace these colors with those easier for them to see, the compensated images could have unnatural color distributions or even artifacts.

There are two major open problems related to compensation of color-weak vision. The first one is that no objective criterion of compensation is available since color perception of an individual is not directly observable or measurable. The second problem is that the degree and characteristics of colorweakness vary widely among individuals and different color stimuli. In order to overcome these difficulties, we proposed earlier a new criterion for colorweakness compensation based on matching between discrimination thresholds of the color-weak observer and of average color-normals. This criterion uses the global theory of Riemann geometry on distance-preserving maps (called isometries) or color-difference-preserving maps. Our target is to compensate such that subjective color differences between every pairs of colors in an image are kept the same for both the color-weak observer and the normal observers. 
The condition in the criterion, taking into account individual characteristics, provides such an isometry between the color spaces of color-normals and described color-weak observer. An implementation of such compensation using local affine transforms is described in [9]. A different approach using geodesic coordinates or Riemann normal coordinates is found in [10] and [11. These compensation methods need many local computations e.g., linear algebraic computation in the first and solution of ODEs in the second approach. One of the reasons is that it is usually hard to express the compensation color explicitly and in a closed form.

In this paper, by applying this criterion to Brettel's color-blind model, we derive a color-weak map for simulation and compensation of color-weak observers along the confusion lines. This isometry map can be described by a parameter called color-blind index, which is defined as the ratio of lengths of discrimination thresholds between the color-weak observer and average color-normals along the confusion line direction. In particular, we obtain an explicit formula of the colorweak map for the simulation and also its inverse map for compensation in closed forms. Therefore the implementation is straightforward and fast. The proposed methods are evaluated by psychological experiments based on the Semantic Differential (SD) method.

\section{Compensation Criterion}

\subsection{Discrimination Thresholds and Color Spaces as Riemann Spaces}

We know that one of the few objectively measurable quantities in color science is the just-noticeable-difference or discrimination thresholds data. Well known examples are the so-called MacAdam ellipses. They define the most important perceptional characteristics of small or local color-differences. At the same time, the discrimination thresholds also provide an excellent representation of individual characteristics for color vision. In fact, these color discrimination thresholds provide the color space with a rich geometric structure which allows us to apply the powerful tools of Riemann geometry.

First of all, the discrimination thresholds provide at every point in a color space a measure of local distance in color space in the following way.

Denote a test color as $x$ and choose it as the origin, for a color vector $y$ in the neighborhood of $x$ with respect to the origin, the discrimination threshold at $x$ can be represented as ellipses(ellipsoids) centered at the test color $x$ :

$$
y^{T} R(x) y=1 \text {. }
$$

Here the positive definite matrix $R(x)$, varying smoothly with the location of the test color $x$, is uniquely determined by the ellipses (ellipsoids) and vice versa.

With such a matrix $R(x)$ defined at every $x$, the local distance around $x$ can be expressed as 


$$
\|d x\|^{2}=d x^{T} R(x) d x
$$

Such a space with a smoothly defined local distance or the matrix $R(x)$ is called a Riemann space, and $R(x)$ defines the Riemann metric 6 . In fact, it has been known since Helmholtz that a color space is a Riemann space which is not Euclidean. Understanding the geometry of this Riemann space and its applications has been an important research fields e.g. 5]. If existing researches mainly used local geometry such as metric tensors, our approach tries to explore the global structure of color spaces.

\subsection{Color Difference Preserving Map and New Criterion of Compensation}

In a Riemann space, the local distance around a point $x$ is defined by the metric $R(x)$, which corresponds to small color difference on a color space. On the other hand, the distance between any two points $x_{1}, x_{2}$ is defined as the length of the shortest curve connecting the two points. The shortest curve between the two points is called the geodesic.

Consider a map $f$ between two color spaces $C_{1}$ and $C_{2}$. Let $R_{1}(x)$ and $R_{1}(y)$ be the Riemann metrics of $C_{1}$ at $x$ and of $C_{2}$ at $y$,

$$
f: C_{1} \longrightarrow C_{2} ; \quad y=f(x)
$$

We wish to preserve the color difference between $C_{1}$ and $C_{2}$, in particular, the distance between any pair $x_{1}$ and $x_{2}$ in $C_{1}$ should be equal to the distance between their images $y_{1}=f\left(x_{1}\right)$ and $y_{2}=f\left(x_{2}\right)$ in $C_{2}$.

In Riemann geometry, a local color-difference preserving map at every point is called a local isometry. A map preserving large color-differences between any pair of colors is a global isometry. In fact, to obtain a local isometry is easy, one only needs to check that the metric $R_{1}(x)$ at every point $x$ is mapped to the metric $R_{2}(y)$ at $y=f(x)$. The global isometry however is hard to deal with, just as large color differences are.

However, it turned out that these two isometries are in fact the same thing. So as soon as one can construct a local isometry, a global one in obtained at the same time.

Assume two color spaces $C_{1}, C_{2}$ describe color-normal observers and a colorweak observer. If we can match the thresholds at every corresponding color pair, such that the small color differences are adjusted to be always the same for every pair, then the large color difference between any corresponding pair of colors is also identical.

Thus, we propose as the criterion of color-weak compensation to transform the color space of the color-weak observer so that it has the same geometry, and therefore the same color differences, as the color space of color-normal observers. The details is given below. 


\subsection{Discrimination Threshold Matching Condition [9]}

We define a color-weak map $w$ which transforms the color space $C_{w}$ of the colorweak observer to $C_{n}$ of color-normal observers as

$$
w: C_{w} \longrightarrow C_{n}: \quad y=w(x)
$$

This map is a local isometry or small color difference preserving if the Jacobian $D_{w}$ of $w$ satisfies the following condition for all $x$ and $y=f(x)$.

$$
R_{n}(y)=D_{w}^{T} R_{w}(x) D_{w}
$$

We also call this equation the threshold matching condition.

Now the compensation map can be defined as the inverse map of the colorweak map $w$, and its local linear approximation is $\left(D_{w}\right)^{-1}$. Applying $w^{-1}$ to the input color image and showing it to color-weak observers will provide them the same experience as the color-normal observer.

\section{Measurement and Estimation of Discrimination Thresholds}

The discrimination thresholds of both the color-normal observers and the colorweak observers are measured using a vision-field-splitting display. The observer sees a square area of $14 \mathrm{~cm} \times 14 \mathrm{~cm}$ from a distance of $80 \mathrm{~cm}$ by using 10 degrees field of vision. Discrimination threshold data were measured from 45 college students (37 male, 8 female, 2 color-weak observers) in CIEXYZ coordinates.

We choose 10 points uniformly sampled within the gamut of the monitor as test colors, and measured thresholds in 14 directions from each test color in CIEXYZ color space. The step-size of the color variation is randomly chosen from $1 \times 10^{-5} \sim 9 \times 10^{-5}$.

To estimate the equations of ellipsoids from these thresholds, we choose the center of the ellipsoid as the origin to eliminate linear terms. Then the 14 sampling points $\mathbf{x}_{i}=\left(x_{i}, y_{i}, z_{i}\right), i=1 \cdots 14$ on the surface of the ellipsoid are substituted into the defining equation. The coefficients in the equation are obtained by least squares fitting.

\subsection{Measurement Results}

The average of thresholds of color-normals and the threshold of the first colorweak observer are shown in Fig 1 and Fig 2 Both ellipses are shown in the u'v' plane Fig 3. It can be observed that the latter is always wider and lie outside of the former, which confirms the reduced sensibility of the color-weak observer. Similar thresholds data of the second color-weak observer are also shown in Fig 4 Fig 5 
The discrimination threshold along the confusion lines of protanopia in $x y$ plane, of the protanopic color-weak observer and average of color-normal observers are shown in Fig 6 .

\section{Compensation Along Confusion Lines}

In the following we apply the method from Section 2 to Brettel's color blind model[1].

This model describes color-blind vision as a projection in LMS space along the $\mathrm{L}$ axis for protanopia and the $\mathrm{M}$ axis for deuteranopia, those axes are in correspondence to the confusion lines. This model consists of the two planes which are spanned by three invariant hues which are perceived equally by both color-normal and color-blind observers.
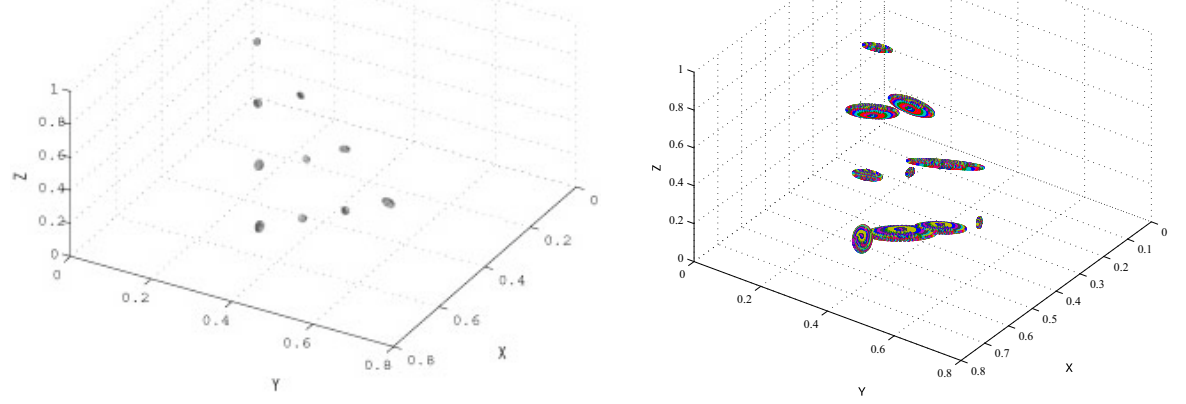

Fig. 1. Threshold ellipsoids of colornormals

Fig. 2. Threshold ellipsoids of the first color-weak

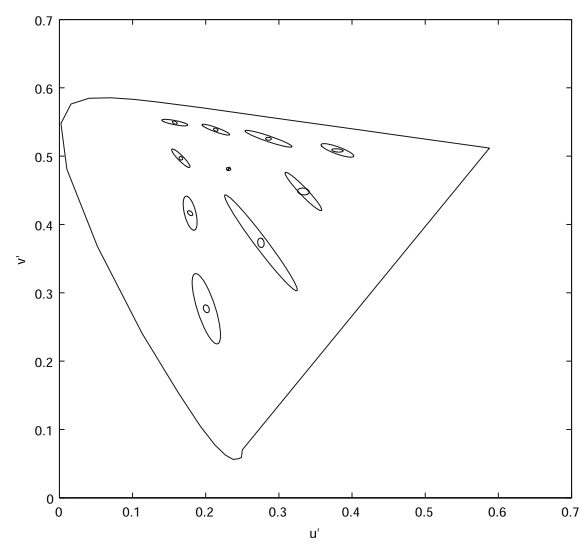

Fig. 3. Ellipses of color-normals and the first color-weak observers on $u^{\prime} v^{\prime}$ plane 


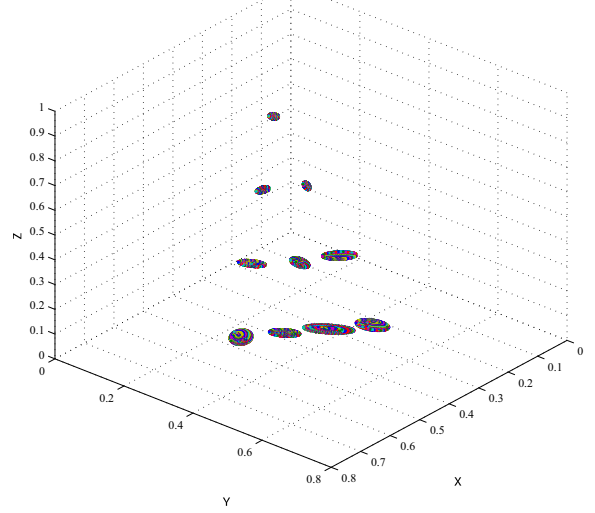

Fig. 4. Threshold ellipsoids of the second color-weak

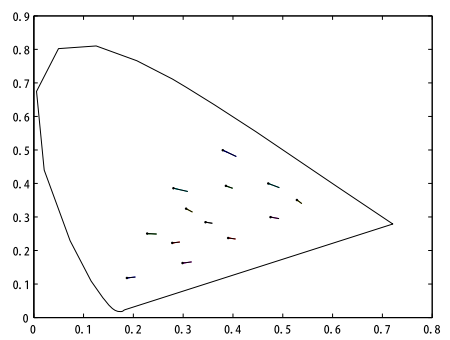

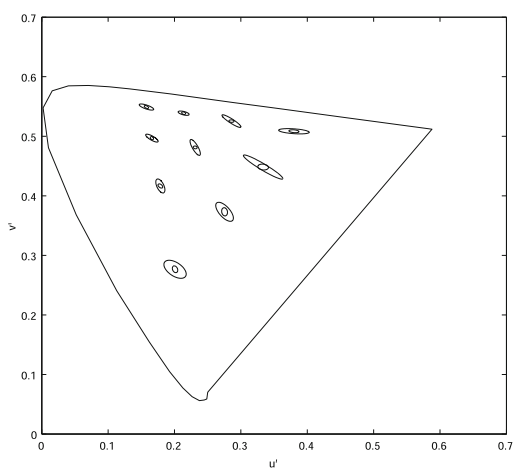

Fig. 5. Ellipsis of the second color-weak on $u^{\prime} v^{\prime}$ plane

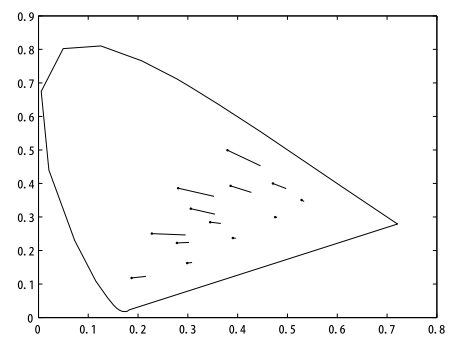

Fig. 6. Thresholds along directions of confusion lines

\subsection{Color-Weak Map and Linear Approximation}

We first consider a linear color-weak model and compensation shown in Fig 7. It is easy to implement and also serves as an intuitive explanation of the rigorous model shown in section 4.2 .

In Fig 7, $\boldsymbol{Q}, \boldsymbol{Q}^{\prime}$ denote the color stimuli perceived by an average color-normal and the color-blind observer. $\boldsymbol{Q}^{\prime}$ is the projection of $Q$ onto the color-blind planes, along $\mathrm{L}$ axis for protanopia and $\mathrm{M}$ axis for deutanopia, corresponding to the confusion lines. Since $\boldsymbol{Q}^{\prime}$ is determined uniquely from $Q$, we use the following notations. For a 3D vector $\boldsymbol{R}$, a scalar $R$ will be used to express position of $\boldsymbol{R}$ on the confusion line passing through it, in term of the distance from $Q^{\prime}$ to $R$.

Linear Color-Weak Map. The color-weak map $w$ maps every stimulus $Q$ toward the color blind plane without reaching it. If we assume $w$ is a linear map, the perception of a color-weak observer defined as $Q^{\prime \prime}$ will be in the form of

$$
Q^{\prime \prime}=w(Q)=\omega Q^{\prime}+(1-\omega) Q \quad(0 \leq \omega<1)
$$




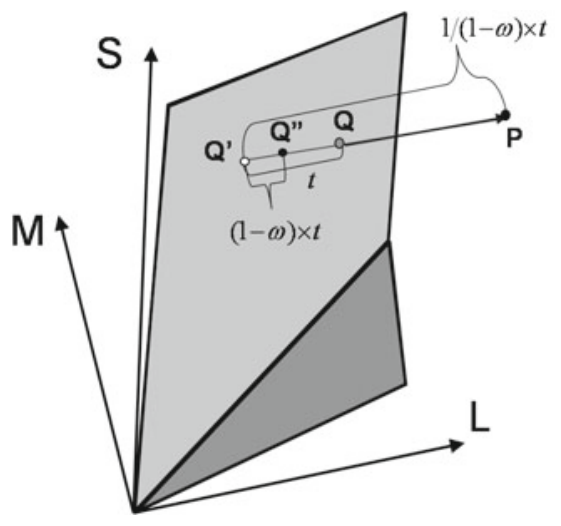

Fig. 7. Linear color-weak model and compensation

Here the parameter $\omega$, which indicates the degree of color-weakness, is called the color-weak index. The observer is completely color-blind if $\omega=1$ and a colornormal if $\omega=0$. Every stimuli $Q$ is mapped along the confusion line to a point $Q^{\prime \prime}$ between $Q$ and color-blind stimulus $Q^{\prime}$, and the distance $Q^{\prime}-Q$ marked as $t$ in Fig 7 is reduced by a factor of $\omega$ at the stop position.

$$
Q^{\prime \prime}=w(Q)=Q+\omega\left(Q^{\prime}-Q\right)
$$

On the other hand, $Q$ moved to $Q^{\prime \prime}$ the reduced distance $\left(Q^{\prime}-Q\right)$ by a factor $1-\omega$.

$$
Q^{\prime \prime}=w(Q)=Q^{\prime}+(1-\omega)\left(Q-Q^{\prime}\right)
$$

Linear Color-Weak Compensation. The color weak map $w$ simulates colorweak vision when applied to the original image. The compensation map is the inverse map $w^{-1}$, which preprocesses the original image as follows.

$$
P=w^{-1}(Q)=Q^{\prime}+\frac{1}{(1-\omega)}\left(Q-Q^{\prime}\right)
$$

Substituting the compensated color $P$ into eq. (44) one can confirm that the colorweak observer actually perceives the same color as color-normals.

Estimation of Color-Weak Index. In order to obtain the color-weak map one needs to estimate the color-weak index $\omega$, which is however not directly observable. In fact, $\omega$ can be uniquely determined by the threshold matching condition in section 2.3. First, the Jacobian of color-weak map $w$ is

$$
D_{w}=1-\omega
$$

Denote the thresholds of color-normal and of the color-weak observer along the confusion line as $\alpha_{n}$ and $\alpha_{w}$. Here the local isometry criterion (3) becomes (9), thus the color-weak index $\omega$ can be defined as (10). 


$$
\begin{gathered}
\alpha_{n}=(1-\omega) \alpha_{w} \\
1-\omega:=\frac{\alpha_{n}}{\alpha_{w}}
\end{gathered}
$$

Notice the statement on linearity of the color-weak map is equivalent to that the color-weak index $\omega$ is a constant on a confusion line. This assumption could be used in implementation to obtain $\omega$ by taking the ratio of the mean on each confusion line of the thresholds of color-normals and of the color-weak observer.

\subsection{Rigorous Definition of Color-Weak Map}

Generally, color-weakness has large variations among different observers and different color stimuli as well. Therefore the color-weak map is not a linear map and $\omega$ is not a constant (see fig 9 ). Below we show a rigorous definition of the color-weak map and compensation.

First, the $1 \mathrm{D}$ color spaces $C_{n}$ for color-normals and $C_{w}$ for the color-weak observer on a confusion line, and the color-weak map $w$ are shown in Fig 8 ,

According to the threshold matching criterion in the section 2.3 , we define the Jacobian of the color-weak map using the color-weak index as follows, here $\omega(Q)$ is the color-weak index at $Q$.

$$
D_{w}(Q)=\frac{\alpha_{n}\left(Q^{\prime \prime}\right)}{\alpha_{w}(Q)}=: 1-\omega(Q)
$$

The color-weak map is then defined by the integral of Jacobian in $C_{w}$ :

$$
Q^{\prime \prime}=w(Q)=Q^{\prime}+\int_{Q^{\prime}}^{Q}(1-\omega(x)) d x
$$

It can be observed to be an extension of (6).

On the other hand, the compensation map can be defined by an integral in $C_{n}$, which is an extension of (77).

$$
P=w^{-1}(Q)=Q^{\prime}+\int_{Q^{\prime}}^{Q} \frac{1}{1-\omega(y)} d y
$$

In fact, one can obtain an exact correspondence between two color spaces of the color-weak observer and color-normals by using the color-weak map (12) and its inverse map (13).

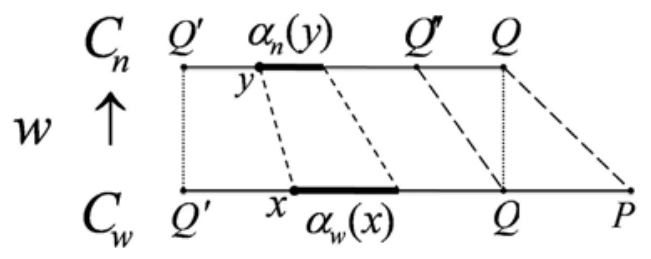

Fig. 8. Rigorous definition of color-weak map 


\subsection{Implementation}

The distribution of the color-weak index $\omega$ on the $u^{\prime} v^{\prime}$ plane is shown in Fig.9. To obtain the color-weak map and the compensation map from the observed discrimination threshold data, we first define the sampling points $\left\{x_{0}, x_{1}, x_{2}, \ldots\right\}$ on the confusion line in $C_{w}$ as follows:

$$
x_{0}=Q^{\prime}, \quad x_{i+1}=x_{i}+\alpha_{w}^{(i)}
$$

Here, $\alpha_{w}^{(i)}:=\alpha_{w}\left(x_{i}\right), i=1,2, \ldots$ are the discrimination thresholds at sampled points $\left\{x_{i}\right\}$.

Similarly, the sampling points $\left\{y_{0}, y_{1}, y_{2}, \ldots\right\}$ on the confusion line of $C_{n}$ are defined using the discrimination threshold data $\alpha_{n}^{(j)}:=\alpha_{n}\left(y_{j}\right), j=1,2, \ldots$ as follows:

$$
y_{0}=Q^{\prime}, \quad y_{j+1}=y_{j}+\alpha_{n}^{(j)}
$$

Then under the color-weak map, one has a precise correspondence between $x_{i}$ and $y_{j}$.

$$
\begin{aligned}
w\left(x_{k}\right) & =Q^{\prime}+\int_{Q^{\prime}}^{x_{k}}(1-\omega(x)) d x=x_{0}+\sum_{i=0}^{k-1} \int_{x_{i}}^{x_{i+1}}(1-\omega(x)) d x \\
& =y_{0}+\sum_{i=0}^{k-1} \alpha_{n}^{(i)}=y_{k}
\end{aligned}
$$

Thus $y_{k}=w\left(x_{k}\right), k=0,1,2, \ldots$, and the color weak index at the $k$-th interval $\omega_{k}$ is defined by using the discrimination threshold of color-normals $\alpha_{n}^{(k)}$ and of the color-weak observer $\alpha_{w}^{(k)}$.

$$
\omega_{k}:=1-\frac{\alpha_{n}^{(k)}}{\alpha_{w}^{(k)}}
$$

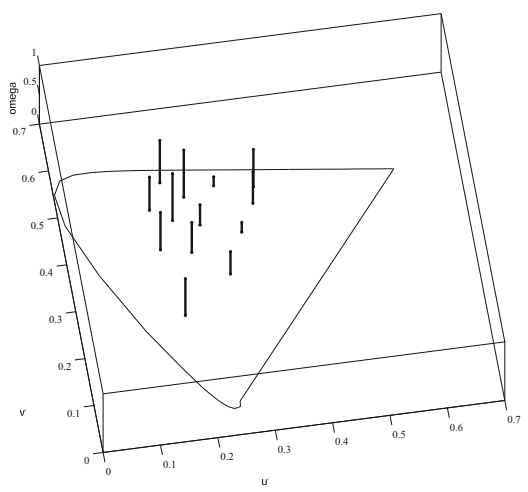

Fig. 9. Distribution of color-weak index 
Assume that $x_{I} \leq Q<x_{I+1}$ in $C_{w}$ and $y_{J} \leq Q<y_{J+1}$ in $C_{n}$.

Assume that the discrimination threshold $\alpha_{w}(x), x \in\left[x_{k-1}, x_{k}\right]$ in the $k$-th interval in $C_{w}$ is a constant equal to that on the right end of the interval $\alpha_{w}^{(k)}$ and the discrimination threshold in $k$-th interval in $C_{n}$ is a constant equal to $\alpha_{n}^{(k)}$, then the color-weak map and the compensation map can be realized by a sum of the discrimination thresholds on the confusion line:

$$
\begin{gathered}
Q^{\prime \prime}=\sum_{i=0}^{I}\left(1-\omega_{i}\right)\left(x_{i+1}-x_{i}\right)=\sum_{i=0}^{I} \alpha_{n}^{(i)} \\
P=\sum_{j=0}^{J} \frac{1}{1-\omega_{j}}\left(y_{j+1}-y_{j}\right)=\sum_{j=0}^{J} \alpha_{w}^{(j)}
\end{gathered}
$$

\section{Simulation}

The color-weak map is applied for the natural image in Fig[11, The color-weak simulated image is shown in Fig 10, the compensated image in Fig[12, Fig[13] and Fig 14 show the change of color distributions in the image before/after compensation in u'v' color space for color-weak observer. It can be observed that for the color-weak observer the distributions have been enlarged. In addition, the area of color distribution is enlarged 1.403 times.

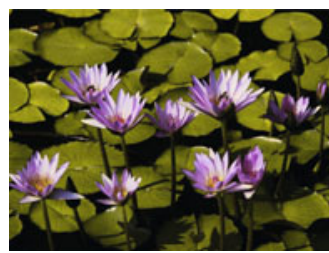

Fig. 10. Simulation

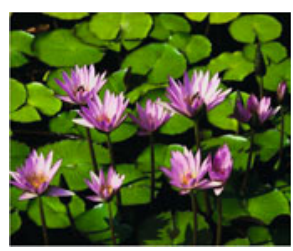

Fig. 11. Original image

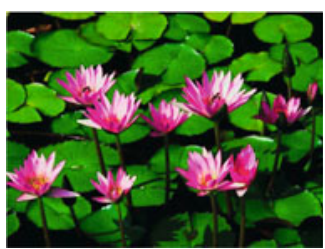

Fig. 12. Compensation

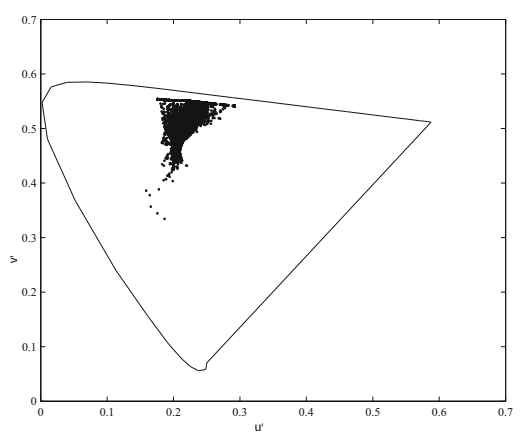

Fig. 13. Color distribution of color-weak simulation image

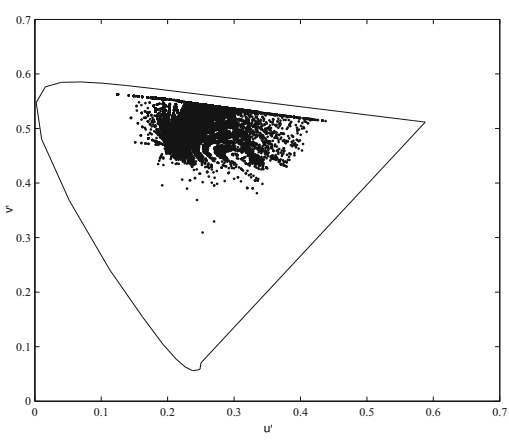

Fig. 14. Color distribution of compensated image 


\section{Evaluation}

We evaluated the compensated images and color-weak simulation images using the semantic differentiation (SD) test. This method is a standard cultureindependent procedure to quantitatively evaluate subjective impressions. The questionnaire about the impression of each image is evaluated using 7 ranks for 8 pairs of opposite adjectives in Fig 15. These 8 pairs of adjectives are selected by another group of 4 color-normals, who are shown 12 images in the SD test and asked their impression in terms of adjectives. The 8 most often mentioned adjective pairs from their answers are chosen as the question adjective pairs in SD test.

Fig 16 shows the original image evaluated by color-normals and the compensated image by the color-weak observer, Fig 17 is the color-weak simulation evaluated by the color-weak observer and the original image evaluated by the color-normal. These comparisons show that the color-weak observer has quite similar impressions from the compensated image as the color-normals from the original image.

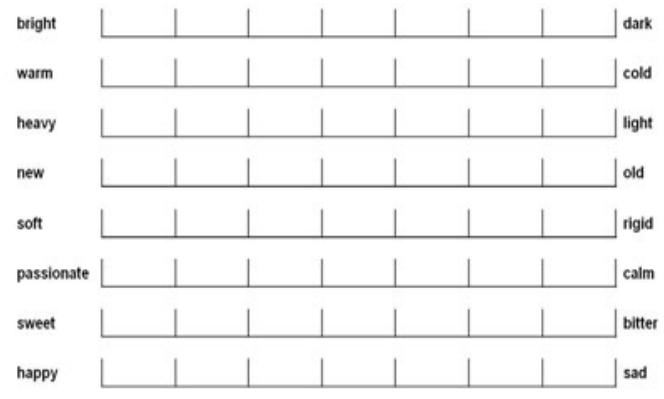

Fig. 15. Questionnaire paper for SD method

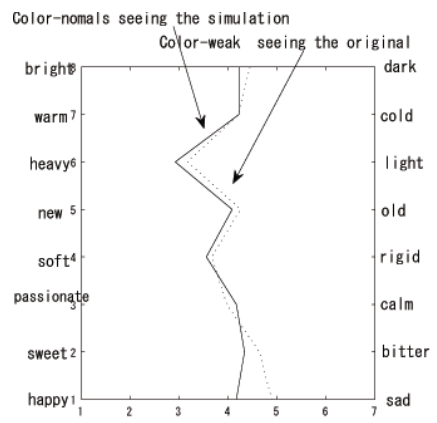

Fig. 16. SD scores before compensation

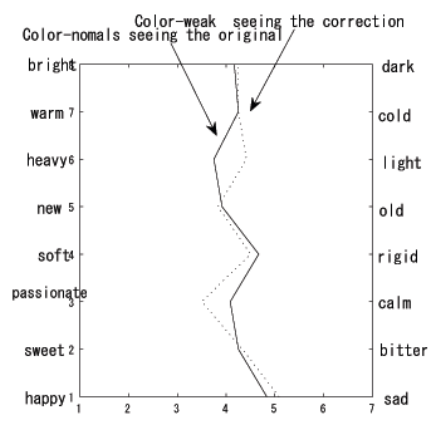

Fig. 17. SD scores after compensation 


\section{Conclusions}

We propose a fast algorithm for color-weak compensation along confusion lines. The compensation is based on the criterion to match discrimination thresholds everywhere between color-normal observers and the color-weak observer. Comparing with previous implementations of compensation with the same criterion, we obtained explicit formulae for color-weak simulation and compensation in a closed form. Hence the new approach is simpler and easier to implement therefore hopefully useful in practice.

Acknowledgement. The authors are grateful to Dr. Reiner Lenz of Linköping Univeristy, Sweden for helpful comments to improve the early version of this paper.

\section{References}

1. Brettel, H., Vienot, F., Mollon, J.D.: Computerized simulation of color appearance for dichromats. Journal of Optical Society of America 14(10), 2647-2655 (1997)

2. Ichikawa, M., Tanaka, K., Kondo, S., Hiroshima, K., Ichikawa, K., Tanabe, S., Fukami, K.: Preliminary Study on Color Modification for Still Images to Realize Barrier-Free Color Vision. In: Proc. IEEE International Conference on Systems, Man and Cybernetics, SMC 2004 (2004)

3. Troiano, L., Birtolo, C., Italiane, P.: Adapting Palettes to Color Vision Deficiencies by Genetic Algorithm. In: Proc. 10th Genetic and Evolutionary Computation Conference in CD-ROM (2008)

4. MacAdam, D.L.: Visual sensitivities to color differences in daylight. Journal of Optical Society of America 32(5), 247-274 (1942)

5. Wyszecki, G., Stiles, W.S.: Color Science, 2nd edn. Wiley Classics Library (2000)

6. do Carmo, M.P.: Riemannian Geometry. Birkhauser, Basel (1992)

7. Chao, J., Osugi, I., Suzuki, M.: On definitions and construction of uniform color space. In: Proceedings of CGIV 2004, The Second European Conference on Colour in Graphics, Imaging and Vision, Aachen, Germany, April 5-8, pp. 55-60 (2004)

8. Regan, B.C., Reffin, J.P., Mollon, J.D.: Luminance noise and the rapid determination of discrimination ellipses in colour deficiency. Vision Research 34(10), 1279-1299 (1994)

9. Mochizuki, R., Nakamura, T., Chao, J., Lenz, R.: Color-weak correction by discrimination threshold matching. In: Proceedings of CGIV 2008, 4th European Conference on Color in Graphics, Imaging, and Vision, Terrassa, Spain, June 9-13, pp. 208-213 (2008)

10. Chao, J., Lenz, R., Matsumoto, D., Nakamura, T.: Riemann geometry for color characterization and mapping. In: Proceedings of CGIV 2008, Proceedings of 4th European Conference on Color in Graphics, Imaging, and Vision, June 9-13, pp. 277-282 (2008)

11. Ohshima, S., Mochizuki, R., Chao, J., Lenz, R.: Color reproduction using riemann normal coordinates. In: Trémeau, A., Schettini, R., Tominaga, S. (eds.) CCIW 2009. LNCS, vol. 5646, pp. 140-149. Springer, Heidelberg (2009) 